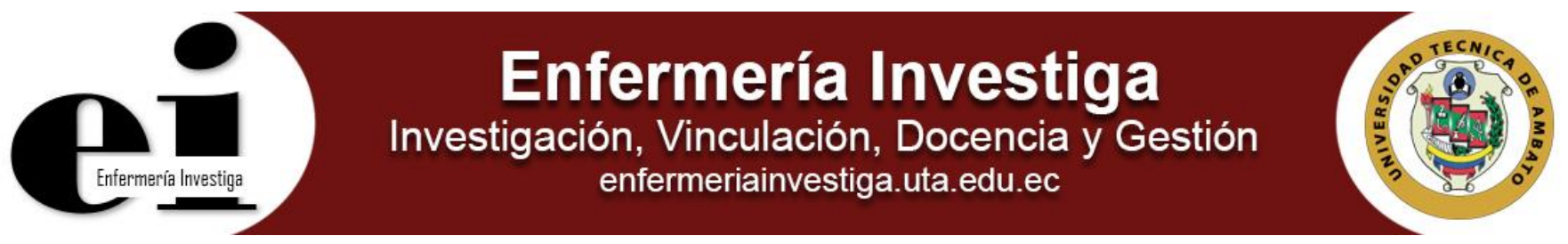

DOI: http://dx.doi.org/10.29033/ei.v3n2.2018.07

Artículo de revisión

\title{
El conocimiento de la Farmacología en el profesional de enfermería
}

The knowledge Pharmacology in nursing professional

\section{Katherine Romero Viamonte ${ }^{1}$}

${ }^{1}$ Universidad Regional Autónoma de los Andes - Facultad de Ciencias Médicas - Carrera de Odontología - Ambato - Ecuador

Romero K. El conocimiento de la Farmacología en el profesional de enfermería. Enferm Inv. 2018;3(2):95-104.

2477-9172 / 2550-6692 Derechos Reservados @ 2018 Universidad Técnica de Ambato, Carrera de Enfermería. Este es un artículo de acceso abierto distribuido bajo los términos de la Licencia Creative Commons, que permite uso ilimitado, distribución y reproducción en cualquier medio, siempre que la obra original es debidamente citada.

\section{Historia:}

Recibido: 16 marzo 2018

Revisado: 13 mayo 2017

Aceptado: 2 junio 2018

Palabras Claves:

Farmacología; conocimiento de la farmacología; enfermería

Keywords: Pharmacology; knowledge of pharmacology; nursing

\begin{abstract}
Resumen
Para lograr una atención médica de calidad, resulta insuficiente realizar el diagnóstico y elegir la terapéutica adecuada. Es cierto que son los primeros aspectos a tomar en cuenta para la cura, alivio y profilaxis de una enfermedad, pero si luego, no se administra el medicamento de la manera adecuada, todo el camino recorrido se vería afectado. El profesional de enfermería juega un importante papel, si toma en consideración aspectos farmacológicos que se encuentran estrechamente vinculados. El presente artículo tuvo como objetivo realizar una puesta al día de los aspectos más relevantes a tener en cuenta por parte de los enfermeros para garantizar la calidad de vida del paciente. La seguridad del medicamento no puede ser un proyecto a largo plazo, ha de ser un desafío constante de cada uno de los profesionales que toca con sus manos el medicamento con el objetivo de mejorar la salud de los pacientes.
\end{abstract}

\begin{abstract}
To achieve quality care is insufficient to diagnose and choose the appropriate treatment. There is no doubt that they are the first things to consider for the cure, mitigation, and even prevention of a particular disease, but certainly not the medication is administered properly, the whole journey will be affected. To do this, the nursing professional plays an important role, if you take into consideration pharmacological aspects that are linked. The objective of this article was to update the most relevant aspects to be taken into account by the nurses in order to guarantee the quality of life of the patient. The safety of the medicine cannot be a long-term project, it has to be a constant challenge in each of the professionals who have in charge the patient's life, in each of the professionals who touches with his hands the medication to be applied an individual with the aim of improving their health and quality of life.
\end{abstract}

Katherine Romero Viamonte. Carrera de Enfermería - Facultad de Ciencias Médicas - Universidad Regional Autónoma de los Andes - Tungurahua - Ambato Ecuador. E-mail: kromeroviamonte@yahoo.es 


\section{Introducción}

En el ámbito de la salud, una de las principales preocupaciones en el siglo XXI es la orientación académica hacia la excelencia, dentro de esta perspectiva es importante no solo el perfil que ofrece la universidad sino también, el modo de enseñanza aprendizaje que el estudiante recibe de parte del docente tanto en la teoría como en la práctica para lograr un estudiante altamente competitivo en beneficio del cuidado integral del paciente. ${ }^{1}$

La farmacología es una de las disciplinas más ligadas a la Enfermería. Un fármaco es considerado como toda sustancia química capaz de inducir una reacción o cambio en el funcionamiento celular y los tejidos vivos.

La farmacología médica, puede entonces definirse como la rama de las ciencias médicas que se ocupa del estudio de los fármacos que se utilizan para el diagnóstico, la prevención o el tratamiento de las enfermedades del ser humano. Más específico, puede definirse también, como la rama de las ciencias biomédicas que estudia; el origen y química de las drogas de utilidad en medicina, sus acciones farmacológicas, sus mecanismos de acción a nivel molecular o celular, la forma como las mismas pasan a través del organismo, sus efectos adversos y sus aplicaciones terapéuticas. ${ }^{2,3}$

Los enfermeros tienen a su cargo una gran diversidad de funciones y tareas, sin embargo, la administración de fármacos forma parte de su haber día tras día por lo que el conocimiento de esta rama es fundamental, además de que en dicha labor, enfrenta un riesgo legal permanente, relacionado con errores en la práctica, dada la responsabilidad y frecuencia con que se realiza.

Del conocimiento adecuado de los fármacos depende la salud, e incluso la vida del paciente, pues a partir de este conocimiento, estos profesionales pueden ser capaces de detectar reacciones adversas, interacciones medicamentosas y errores de medicación, además, garantiza una administración eficaz y segura, y le permite orientar al paciente sobre el consumo de los mismos.

El aprendizaje de la farmacología puede resultar complejo, especialmente en los estudiantes pues, consideran que implica mucha memorización de datos; por lo que resulta recomendable trabajar con la ayuda de casos prácticos, situaciones reales o no, que ocurren a un paciente problema con el que se va a trabajar. De esta forma es más fácil retener dosis, cantidades, aspectos, etc. Otra forma de estudio es trabajar los fármacos clasificándolos según la parte del cuerpo en la que actúan, que es la forma más habitual de encontrar los manuales farmacológicos. ${ }^{4}$
A partir de lo expuesto, el objetivo del presente artículo es realizar una puesta al día de los aspectos más relevantes a tener en cuenta por parte del personal de enfermería al momento de la administración de medicamentos.

\section{Desarrollo}

Todas las sociedades del mundo, ligan su desarrollo a la capacidad biológica del hombre, de ahí que el deber fundamental de los gobiernos es proteger la salud de todos los ciudadanos, o recuperarla cuando se altera.

La importancia del conocimiento de esta rama de las ciencias médicas, la farmacología, puede fácilmente comprenderse si se considera que, en su relación profesional con el paciente, el médico debe resolver en definitiva dos únicos y fundamentales problemas: hacer el diagnóstico y establecer la mejor terapéutica, razón por la cual es ineludible la importancia del conocimiento de la Farmacología por parte del médico pero, ¿acaso esto sería suficiente para lograr los verdaderos propósitos sobre la salud humana? El binomio médico - enfermero es imposible de disolver en todos los aspectos desde el punto de vista laboral.

A consideración de los autores del presente artículo, para lograr una atención con calidad, resulta insuficiente realizar un diagnóstico y elegir la terapéutica adecuada. No cabe dudas de que son los primeros aspectos a tomar en cuenta para la cura, alivio, e incluso profilaxis de una enfermedad determinada, pero si luego, no se administra el medicamento de la manera adecuada, todo el camino recorrido se vería afectado, este es el inicio del análisis del tan importante papel del conocimiento de la Farmacología por parte del personal de enfermería.

El Proceso de Atención de Enfermería (PAE) constituye la estrategia de actuación del enfermero para brindarle al paciente un cuidado total. Se basa en la aplicación del método científico y consiste en una serie de acciones señaladas y pensadas para cumplir con el objetivo de la enfermería. Dentro del PAE, existen cinco etapas que muy bien pueden vincularse con la farmacología: valoración, diagnóstico, planificación, ejecución y evaluación. ${ }^{5,6}$

Las fases del proceso enfermero están ordenadas, estrechamente vinculadas y son correlativas por lo que, afecta de manera directa al resto, conteniendo a su vez diferentes pasos (Figura 1). ${ }^{7}$ 
Figura 1. Fases del proceso enfermero

\begin{tabular}{|c|c|}
\hline Valoración & $\begin{array}{l}\text { - Obtención de datos } \\
\text { - Validación de datos } \\
\text { - Organización de la información }\end{array}$ \\
\hline Diagnóstico & $\begin{array}{l}\text { - Análisis de datos } \\
\text { - Identificación de problemas } \\
\text { - Formulación de problemas }\end{array}$ \\
\hline Planificación & $\begin{array}{l}\text { - Priorización de problemas } \\
\text { - Establecimiento de objetivos } \\
\text { - Selección de acciones }\end{array}$ \\
\hline Ejecución & $\begin{array}{l}\text { - Favorecer la relacion de ayuda } \\
\text { - Planificación de la accion }\end{array}$ \\
\hline Evaluación & $\begin{array}{l}\text { - Valoracion del paciente } \\
\text { - Comparacion dedatos actuales } \\
\text { con los objetivos } \\
\text { - Modificación del plan (si se } \\
\text { precisa) }\end{array}$ \\
\hline
\end{tabular}

Fuente: Manual CTO Oposiciones de enfermería.

Durante la etapa de valoración, el enfermero realiza una comprobación de la prescripción realizada por el médico, revisa la historia clínica del paciente y comprueba por este medio y a través de la anamnesis si el paciente tiene antecedentes de alergia a algún medicamento incluido el prescrito y valora la vía de administración a utilizar y las posibles dificultades.

Durante el diagnóstico de enfermería, el enfermero determina si existe incumplimiento o manejo ineficaz de la terapia por parte de los familiares o del propio paciente y determina además, de forma precoz la aparición de reacciones adversas e interacciones medicamentosas o con los alimentos.

En la etapa de planificación, el enfermero realiza el cálculo de la dosis del medicamento a administrar, analiza cómo y con qué debe preparar el medicamento y permeabiliza la vía.

La etapa de ejecución incluye la administración del medicamento por la vía adecuada y la educación a pacientes y familiares acerca del tema.

$Y$ en la etapa de evaluación, el enfermero comprueba si se han alcanzado los objetivos terapéuticos y comunica al personal indicado. Por supuesto, para poder reconocer si la respuesta fue adecuada o no, debe tener conocimientos amplios acerca de los efectos terapéuticos o adversos de los medicamentos. $^{8}$

Pero veamos algunos de estos aspectos farmacológicos, vinculados todos entre sí: administración de medicamentos, detección de reacciones adversas, detección de interacciones medicamentosas, errores de medicación y educación al paciente.

\section{Vías de administración de medicamentos}

Al hablar de vías de administración, se hace referencia a las distintas formas o medios por las cuales se hacen llegar los medicamentos al organismo, las mismas varían en dependencia de si desea o no una acción en el sitio donde se aplique o si se desea una acción sistémica luego de administrado el medicamento. ${ }^{9}$

El enfermero es el personal indicado para la administración de medicamentos, en su haber está el conocimiento de las diferentes vías en que se administran, conocimiento que va más allá del sitio de inyección o del sitio donde se coloca el medicamento, es necesario que conozca además, las desventajas y ventajas que aportan cada una de estas vías y cuál es la más biodisponible en dependencia de las características individuales de cada paciente.

La administración segura del medicamento incluye además, la dosis que se va a administrar, los horarios y la frecuencia correcta y por supuesto el paciente correcto.

Para garantizar una administración segura de medicamentos, existe una regla nemotécnica, denominada "los nueve correctos", que aunque no garantiza que no se produzcan errores en la administración, si es cierto que se pueden prevenir los errores de manera significativa y puede mejorar la seguridad y la calidad de la atención prestada al paciente durante el proceso de administración de medicamentos ${ }^{10}$ : paciente correcto. -Medicamento correcto. -Vía correcta. -Horario correcto. -Dosis correcta. -Registro correcto. -Acción correcta. -Forma correcta. -Respuesta correcta.

Es importante resaltar que según la literatura consultada, se puede hacer referencia a nueve correctos, 10 correctos e incluso, 5 correctos.

El enfermero que carezca de este tipo de conocimiento de la farmacología pone en riesgo la vida del paciente. El éxito de la terapéutica se consolida gracias a la pericia profesional del personal de enfermería y para ello necesita de los conocimientos de la Farmacología pues cada uno de los medicamentos, según su presentación, requiere un método diferente de preparación y administración, de esta manera podrá actuar de forma segura ante cada paciente en cuestión.

Lamentablemente, estos problemas persisten y se considera que la base se encuentra en la formación del estudiante de Enfermería.

En un estudio realizado por Tovar y Saavedra se encontró que, a pesar de manifestar los estudiantes de 5 to semestre de enfermería que tienen estrategias de estudio como lecturas autorreguladas, tutorías presenciales y ayudas audiovisuales; 
cuentan con mala capacidad para recordar lo revisado, consideran la materia de farmacología con mayor dificultad para el aprendizaje, el aprendizaje es solo memorístico no recuerdan lo anterior explicado por lo que no se consideran con suficientes conocimientos para la administración segura de medicamentos desde la interpretación hasta los efectos adversos. ${ }^{11}$

Llama la atención en un estudio realizado en fecha reciente, que los médicos presentaban un nivel de conocimiento mayor que los enfermeros en el tema de administración segura de medicamentos, cuando, como ya se ha explicado hasta aquí, que son estos últimos los máximos responsables de esta labor. ${ }^{10}$ En la actualidad esta práctica ha sido delegada al enfermero siempre y cuando el médico lo prescriba.

\section{Reacciones Adversas a Medicamentos}

Otro aspecto a considerar es la detección de reacciones adversas a medicamentos (RAM). Cada vez hay más pruebas de que las RAM son una causa frecuente, aunque a menudo prevenible, de enfermedad, discapacidad o incluso muerte, hasta el punto de que en algunos países figuran entre las 10 causas principales de mortalidad. ${ }^{12}$

Esta definición es atribuida a toda respuesta nociva y no intencionada que se produce tras la administración de un medicamento a dosis utilizadas normalmente por el hombre, con el objetivo de curar, prevenir o diagnosticar una enfermedad determinada, por lo que queda excluida de este planteamiento la sobredosis.

En la actualidad, la Organización Mundial de la Salud (OMS), ha ampliado el concepto de farmacovigilancia. La define como: "La disciplina que trata de la recolección, seguimiento, investigación, valoración y evaluación de la información procedente de los profesionales de la salud y de los pacientes, sobre reacciones adversas a los medicamentos, productos bilógicos, plantas medicinales y medicinas tradicionales, con el objetivo de identificar nuevos datos sobre riesgos y prevenir daños sobre el paciente. ${ }^{13}$

A través de esta nueva definición queda claro que el personal de enfermería juega un papel fundamental en la detección de reacciones adversas, más, cuando es el personal que está la mayor parte del tiempo al lado del paciente.

Gracias a la farmacología, el personal de enfermería adquiere los conocimientos y habilidades para detectar cualquier acontecimiento adverso, en su formación está recibir de cada grupo farmacológico, sus acciones o efectos terapéuticos pero también, sus efectos indeseables, de esta manera sería capaz de reconocer en la práctica esta eventualidad y actuar en consecuencia. Sin el conocimiento previo de esta temática, el paciente correría un riesgo aun mayor al pensarse, por ejemplo, que es un nuevo síntoma que aparece y no se tome la conducta adecuada.

En un estudio sobre conocimiento del manejo práctico de fármacos en enfermería se determinó que a pesar de que la mayoría conocía los medicamentos que se utilizaron en el servicio investigado y dominaron la administración de los mismos, el $62.5 \%$ no era capaz de identificar las reacciones adversas. ${ }^{14}$

En otra investigación se demostró que el $46.5 \%$ del personal de enfermería entrevistado en el hospital, mencionó que el tema de RAM nunca había sido abordado en la institución y un $20.9 \%$ manifestó que el conocimiento ya se había discutido pero de forma insuficiente. Al cuestionar sobre los factores que dificultan la identificación de las RAM en el paciente pediátrico y/o neonatal, se verificó que los dos más citados fueron: esquema terapéutico en que son administrados varios fármacos de forma simultánea y desconocimiento por parte del profesional de salud, acerca de las RAM. ${ }^{15}$

En Ecuador, el Sistema Nacional de Farmacovigilancia fue creado en el año 2011 con el fin de recopilar, evaluar, codificar, analizar, registrar y comunicar cualquier sospecha de reacción adversa a algún medicamento que se comercializa en el país, y en estos momentos se están formando a los profesionales sanitarios sobre él. ${ }^{16}$ Sin embargo, muchos otros países, van a la vanguardia en esta importante área del mundo de los medicamentos.

Todo profesional de la salud, incluidos enfermeros, deben sensibilizarse con que, el reporte de las reacciones adversas no es una simple actividad estadística, sino que en ello va implícito la vida del paciente.

Con sus más de 50 años de historia, la farmacovigilancia sigue siendo una disciplina científica y clínica muy dinámica, resulta imprescindible para afrontar los problemas que pueda plantear un arsenal medicamentoso que no deja de crecer en variedad y potencia, porque todo fármaco encierra un inevitable y a veces impredecible potencial dañino. Por eso es tan necesario que, en cuanto surjan efectos adversos o toxicidad (sobre todo si aún no están descritos), se notifique y analice el episodio y se comunique de forma adecuada su importancia a un público que sepa cómo interpretar la información. ${ }^{13}$

\section{Interacciones medicamentosas}

La interacción medicamentosa es considerada un fenómeno que ocurre cuando los efectos de un fármaco son modificados debido a la administración simultánea de otro fármaco o alimento, dando como resultado disminución, anulación o aumento del efecto de uno de los fármacos. ${ }^{17}$ 
El hecho de que el número de interacciones medicamentosas ocurridas en la práctica clínica sea pequeño en comparación con las que aparecen descritas en la literatura, no es razón para restarle importancia al tema, y existen motivos varios, como, pérdida considerable de la acción terapéutica, que de dicha interacción se pueda suponer el éxito o el fracaso del tratamiento, o que se formen otras sustancias que no sean de interés para el tratamiento; razones todas que pueden poner en riesgo la vida del paciente.

Bien es cierto que muchas de estas interacciones son inevitables, fundamentalmente en los pacientes hospitalizados, los cuales durante este periodo pueden consumir hasta siete medicamentos por día, pero gran parte de las interacciones también pueden ser evitadas, en particular, aquellas que están estrechamente relacionadas con el personal de enfermería: incompatibilidad, reconstitución, dilución y errores de medicación. Al respecto, se han realizado algunos avances para determinar cuáles son los antecedentes de tratamiento farmacológico y las combinaciones de fármacos particularmente problemáticos, con el fin de establecer distintos niveles de riesgo y también, métodos para reducirlos, entre otros la supresión de algunos medicamentos prescritos, como se ha hecho en el Canadá y en los Estados Unidos de América. Sin embargo, estas iniciativas solucionan solamente una parte del problema de la polimedicación. ${ }^{18}$

En el momento de la administración de los medicamentos, el enfermero debe poner en práctica los conocimientos adquiridos en farmacología, relacionados con las interacciones medicamentosas, la conocida interacción entre penicilina-gentamicina es un ejemplo de ello, esta es una de las combinaciones más utilizadas en la práctica médica con el objetivo de obtenerse un efecto sinérgico, sin embargo, si se preparan en la misma jeringa ocurre una incompatibilidad físico química por lo que no se lograría el efecto deseado. ${ }^{19}$

La reconstitución de los medicamentos para su administración también resulta de vital importancia. Se habla de reconstitución cuando se le administra agua u otro solvente a un medicamento liofilizado. A través de la farmacología, el enfermero recibe los conocimientos relacionados con el tema, sabrá qué volumen de medicamento administrar según la viscosidad del mismo y el sitio de inyección, las técnicas de asepsia a tener en cuenta, verificar si existe turbiedad, cambio de coloración o formación de partículas sólidas. El enfermero también aprenderá, para su posterior aplicación con el paciente, que las soluciones con aditivos deben ser preparadas inmediatamente, si son soluciones fotosensibles deberán ser protegidas de la luz debidamente y que no todos los medicamentos se pueden reconstituir 0 diluir con las mismas sustancias. $^{20}$
Por otro lado, hacer coincidir la ingesta de medicamentos con el horario de las comidas es muy frecuente, sin embargo, este método a pesar de que resulta ventajoso, en otros casos, puede suceder lo contrario. Hacer coincidir el medicamento con alimentos, permite que el paciente degluta estos últimos más fácilmente, existe mejor tolerancia gástrica y facilita el cumplimiento de los horarios, y en algunos casos, permite que se aumente la absorción o efecto de los fármacos, sin embargo, existen medicamentos donde ocurre todo lo contrario, o sea, la absorción o acción de estas sustancias se ve interferida por la concomitancia con los alimentos.

Poco a poco las interacciones fármaco-nutrimento han ido cobrando importancia, mientras hace algunos años no eran tomadas en cuenta; no obstante, en la actualidad se sabe que los alimentos contienen nutrimentos y otras sustancias que pueden afectar en diferente grado la farmacocinética y la farmacodinamia de los medicamentos $y$, de igual manera, los medicamentos causan impacto sobre el estado nutricional, ya sea produciendo una deficiencia nutricional 0 interfiriendo en el proceso natural de la alimentación (generando hambre o anorexia, vómitos o náuseas, etcétera). Estas interacciones, además de ser bidireccionales, pueden ser desde leves hasta muy graves, e incluso mortales, por lo que no deben subestimarse. ${ }^{20}$

De que toda esta información llegue al paciente para que puedan lograrse los efectos terapéuticos, es responsable, en gran medida, el personal de enfermería, él será el encargado de transmitir esta información al paciente ambulatorio, o de aplicarlo de la manera adecuada en el paciente hospitalizado, pero para ello, debe estar preparado desde el punto de vista farmacológico, conocer qué medicamentos son convenientes administrarlos de conjunto con las comidas y cuáles no, información que recibirá a través de la asignatura farmacología y con su actualización constante sobre esta ciencia.

En una investigación realizada en un hospital de Brasil el $80.0 \%$ de los enfermeros encuestados manifestó que tenían conocimiento para detectar apenas algunas interacciones medicamentosas por lo que no se garantizaba una práctica segura en la administración de medicamentos. ${ }^{21}$

\section{Errores de medicación}

Bien es cierto que los medicamentos han sido creados para causar un beneficio en el ser humano, sin embargo, el uso incorrecto de ellos o la omisión en su administración podrían tener un efecto mortal.

Durante muchos años la literatura profesional ha descrito los problemas relacionados con los medicamentos, pero estos aún persisten. Ya desde el año 1999 existen reportes del Instituto Nacional de 
Medicina de los Estados Unidos donde se plantea que ocurren alrededor de 44000 a 98000 muertes anuales en el país a causa de errores en la medicación. ${ }^{22}$

Aun cuando el tema no es nuevo, persiste una cierta confusión sobre los términos utilizados para denominar a los distintos efectos negativos producidos por los medicamentos, lo cual dificulta en gran medida el conocimiento de la incidencia real de dichos efectos y la comparación de los resultados obtenidos en los diferentes estudios. Por ello, resulta conveniente aclarar dichos términos, aunque es preciso señalar que no hay un consenso al respecto.

Error de medicación (EM) es cualquier error que se produce en cualquiera de los procesos del sistema de utilización de los medicamentos. El National Coordinating Council for Medication Error Reporting and Prevention (NCCMERP) define los EM como: "cualquier incidente prevenible que puede causar daño al paciente o dar lugar a una utilización inapropiada de los medicamentos, cuando éstos están bajo el control de los profesionales sanitarios o del paciente o consumidor. Estos incidentes pueden estar relacionados con la práctica profesional, con los productos, con los procedimientos o con los sistemas, incluyendo fallos en la prescripción, comunicación, etiquetado, envasado, denominación, preparación, dispensación, distribución, administración, educación, seguimiento y utilización". ${ }^{23}$

El hecho de que se produzca un EM no indica necesariamente que deba producirse un daño en el paciente (acontecimiento adverso).

Un acontecimiento adverso potencial es un EM grave que podría haber causado un daño, pero que no lo llegó a causar, bien por suerte (por ejemplo, el paciente no tuvo una reacción alérgica a un medicamento que recibió, a pesar de que estaba anotado en la historia clínica que sí era alérgico) o bien porque fue interceptado antes de que llegara al paciente (por ejemplo, la enfermera se dio cuenta de que estaba prescrito un medicamento al que el paciente era alérgico y contactó con el médico para que lo cambiara) ${ }^{24}$

Un acontecimiento adverso por medicamentos (AAM) se define como "cualquier daño, grave o leve, causado por el uso (incluyendo la falta de uso) de un medicamento" o "cualquier daño resultante del uso clínico de un medicamento". Los AAM pueden clasificarse en dos tipos: AAM prevenibles (son aquellos causados por EM. Suponen, por tanto, daño y error) y AAM no prevenibles (son aquellos que se producen a pesar de un uso apropiado de los medicamentos (daño sin error) y se corresponden con las denominadas reacciones adversas a medicamentos (RAM). ${ }^{25}$
El análisis de los acontecimientos adversos por medicamentos (AAM) potenciales es útil, ya que permite identificar tanto los puntos donde falla el sistema y se producen los errores, como los puntos donde funciona y los errores se consiguen interceptar y evitar. ${ }^{24}$

Los errores de medicación se clasifican en distintos tipos según la naturaleza de estos.

En 1998 el NCCMERP publicó la primera taxonomía de errores de medicación con el fin de proporcionar un lenguaje estandarizado y una clasificación estructurada de los mismos, para su posterior análisis y registro (Tabla 1). ${ }^{23,26}$

Si bien es cierto que la información mostrada a continuación resulta de gran utilidad para clasificar los errores de medicación, resulta de gran importancia conocer también, por qué sucedieron.

Claramente se observa que el problema es multifactorial, donde, a partir de la clasificaciones, el enfermero juega un papel fundamental a la hora de evitar los errores de medicación, pero la principal manera de prevenirlos es a través de los conocimientos de la farmacología, pues resulta evidente que la gran mayoría de ellos son prevenibles.

Son varios los estudios en Ecuador que demuestran los fallos relacionados con la medicación y que en ellos se encuentra involucrado el profesional de Enfermería. En un estudio realizado en un hospital de la provincia de Imbabura, se determinó que, en un periodo de 30 días se detectaron 26 eventos adversos relacionados con los cuidados de enfermería. Aunque el $50 \%$ se tipificó como suceso sin lesión, el $100 \%$ fue clasificado como potencialmente prevenible. La mayoría estuvo relacionado con mala técnica en el momento de administrar el medicamento, incumplimiento del horario e incumplimiento de las indicaciones médicas. $^{27}$

En otro hospital del país se evidenció que el $86 \%$ de los profesionales de enfermería incurrieron en errores con respecto a alterar la frecuencia en la administración de medicamentos, enfatizando que si no se administra el medicamento a la hora y dosis correcta, no se logra la acción y efecto deseado de los medicamentos, lo que aumenta la estadía hospitalaria, razón por la que se exige para el profesional de enfermería, conocimiento científico, técnico y ético para cumplir con seguridad esta actividad de gran importancia. ${ }^{28}$

Válido aclarar que esta problemática persiste a nivel mundial, se menciona el caso de Chile donde, en un hospital, se detectaron un total de 625 incidentes de seguridad del paciente con una media de 0,7 incidentes por paciente y los errores de medicación 
representaron el 89,56\% $(n=558)$ del total de incidentes. Los autores, a diferencia de muchos, hacen alusión a que los errores de medicación no estuvieron correlacionados con la carga laboral del profesional de enfermería lo que sugiere que su ocurrencia también pudo estar influenciada por factores no estudiados en esta investigación y donde mencionan como posibles, las fallas activas, el grado de adhesión a protocolos, la supervisión, entre otros. ${ }^{29}$

Tabla 1. Tipos de errores. Clasificación del NCCMERP $(23,26)$

\section{Medicamento erróneo}

\subsection{Selección inapropiada del medicamento}

1.1.1. Medicamento no indicado/apropiado para el diagnóstico que se pretende tratar

1.1.2. Historia previa de alergia o efecto adverso similar con el mismo medicamento o con otros similares

\subsubsection{Medicamento contraindicado}

1.1.4. Medicamento inapropiado para el paciente por su edad, situación clínica o enfermedad subyacente

\subsubsection{Duplicidad terapéutica}

\subsection{Medicamento innecesario}

1.3. Transcripción/dispensación/administración de un medicamento diferente al prescrito

\section{Omisión de dosis o de medicamento}

2.1. Falta de prescripción de un medicamento necesario

\subsection{Omisión en la transcripción}

2.3. Omisión en la dispensación

2.4. Omisión en la administración

\section{Dosis incorrecta}

3.1. Dosis mayor de la correcta

3.2. Dosis menor de la correcta

\subsection{Dosis extra}

4. Frecuencia de administración errónea

5. Forma farmacéutica errónea

6. Error de preparación, manipulación y/o
acondicionamiento

7. Técnica de administración incorrecta

8. Vía de administración errónea

9. Velocidad de administración errónea

10. Hora de administración incorrecta

11. Paciente equivocado

12. Duración del tratamiento incorrecta

12.1. Duración mayor de la correcta

12.2. Duración menor de la correcta

13. Monitorización insuficiente del tratamiento

13.1. Falta de revisión clínica

\subsection{Falta de controles analíticos}

13.3. Interacción medicamento-medicamento

13.4. Interacción medicamento-alimento

14. Medicamento deteriorado

15. Falta de cumplimiento por el paciente

16. Otros

\section{Educación al paciente}

Aunque parezca distante del tema tratado, otro aspecto de relevante importancia es la educación al paciente por parte del profesional de enfermería.

En el año 1970, se publica el informe técnico 446 de la OMS, "Farmacología Clínica: actividades, servicios y enseñanza", en el que se recomienda el desarrollo de la especialidad como una disciplina integrada en los sistemas de salud, y en el que se señala entre sus funciones "mejorar el cuidado de los pacientes promoviendo un uso más efectivo y seguro de los medicamentos, incrementar el conocimiento a través de la investigación, transmitir este conocimiento a través de la enseñanza y promover servicios tales como información sobre medicamentos, análisis de fármacos, monitorización del abuso de fármacos y asesoría en el diseño de estudios". ${ }^{30}$

Se pretende resaltar de la mencionada definición aspectos como, "disciplina integrada", "promoción de uso efectivo de medicamentos", "transmitir conocimientos sobre medicamentos", "promover información sobre uso de los medicamentos" todo ello con el fin de "mejorar el cuidado de los pacientes". La única manera de poder cumplir con lo establecido por la OMS, es a través de un conocimiento pleno de la farmacología, de otra forma es imposible pretender orientar al paciente acerca de cómo o cuál es la mejor manera de utilizar los medicamentos y que preserve su salud.

Es imposible deslindar el tratamiento farmacológico y el no farmacológico para una enfermedad determinada, ambos se complementan sustancialmente. Es en los libros de farmacología donde se muestra dicha información y es el profesional de enfermería el personal encargado de orientar y educar al paciente acerca del cumplimiento del mismo.

Según la OMS, la educación para la salud puede definirse desde dos vertientes. Por una parte, consiste en proporcionar a la población los conocimientos, habilidades y destrezas necesarias para la promoción y la protección de la salud. Por otro lado, la educación para la salud contribuye a capacitar a los individuos para que participen activamente en definir sus necesidades y elaborar propuestas para conseguir unas determinadas metas en salud. ${ }^{31}$ 
Para mejorar la salud de las personas es necesario que los individuos aumenten sus conocimientos sobre la salud y la enfermedad, sobre el cuerpo y sus funciones, utilidad de los Servicios de Salud y factores que actúan sobre la salud.

El personal de enfermería utiliza, junto con otros miembros del equipo de salud, dos actividades fundamentalmente, las charlas educativas y las demostraciones grupales. Teniendo en cuenta que, es la relación con el usuario, la principal actividad de cualquier miembro del equipo, tanto en el domicilio como en el centro sanitario.

La enfermería abarca los cuidados, autónomos y en colaboración, que se prestan a las personas de todas las edades, familias, grupos y comunidades, enfermos o sanos, en todos los contextos, e incluye la promoción de la salud, la prevención de la enfermedad, y los cuidados de los enfermos, discapacitados, y personas moribundas. Funciones esenciales de la enfermería son la defensa, el fomento de un entorno seguro, la investigación, la participación en la política de salud y en la gestión de los pacientes y los sistemas de salud, y la formación. ${ }^{32,33}$

Los problemas de salud son distintos para cada grupo de edad. En el individuo joven, la principal causa de muerte, son los accidentes, que provocan una disminución de la fuerza de trabajo, creando cierto grado de dependencia en el caso de pacientes hospitalizados.

Otro de los problemas que con gran frecuencia se presentan es la enfermedad aguda, como por ejemplo: la infección del aparato respiratorio superior; pero es con el estrés con el que se desarrollan las úlceras gástricas y duodenales, pudiéndose llegar a conductas autodestructivas, como por ejemplo el abuso de drogas, alcoholismo o suicidio, que junto con los accidentes ya citados anteriormente comprenden las principales causas de muerte en el adulto joven. ${ }^{34}$

En el adulto de mediana edad, las principales formas de enfermar son las afecciones cardiovasculares, y pulmonares, artritis reumatoide, cáncer, diabetes mellitus, obesidad, alcoholismo, ansiedad y depresión; la mayoría de las cuales produce con frecuencia ingreso en unidades de atención especializada.

En el anciano, las formas de enfermar tienen características peculiares, incrementándose las enfermedades de origen degenerativo como son las enfermedades cardiacas, cáncer, enfermedad pulmonar obstructiva crónica, neumonía, edema pulmonar, enfermedad vascular, artritis, enfermedades cutáneas, y accidentes (caídas, quemaduras, intoxicaciones); causas todas ellas de largos periodos de hospitalización. Otras afecciones comunes de la vejez son la pérdida de audición, cataratas y errores de refracción, dolores osteoraticulares, con la particularidad de que, a medida que se envejece aumenta la probabilidad de experimentar varias afecciones al mismo tiempo. ${ }^{35}$

La gestación también es una etapa de la vida de la mujer y de la pareja donde se necesita educación, la psicoprofilaxis del parto ha contribuido mucho hoy en día a que se obtengan mejores resultados a la hora del nacimiento del bebé

Todos estos grupos, según sus características, necesitan de educación para la salud, más, cuando muchos de estos procesos tienden a ser crónicos como la Diabetes Mellitus y las enfermedades cardiovasculares y que provoca en gran medica que el paciente abandone el tratamiento o el estilo de vida saludable.

A través de estos ejemplos se observa claramente la utilidad del personal de enfermería, cuya profesión se encuentra orientada a brindar cuidados de atención directa al paciente, bien para que recobre su estado de salud, bien para adaptarse a las secuelas de alguna enfermedad padecida o bien en aras de evitar complicaciones.

En una investigación realizada en Colombia en lo referente al tema y cuyo objetivo fue explorar el efecto de una intervención de enfermería en la administración de medicamentos a niños con enfermedades respiratorias se obtuvo que el grupo experimental tuvo diferencias en cuanto a ganancia de peso, días de estancia hospitalaria, comprensión de padres sobre la enseñanza del uso seguro de la medicación, entre otras variables con respecto a los niños que no recibieron la intervención, lo que permitió demostrar que el proceso de enfermería es útil en la formación académica dando identidad al ejercicio profesional. Con la intervención se promueve la administración correcta y segura de medicamentos, a la vez que contribuye a la práctica de enfermería basada en la evidencia. ${ }^{36}$

El personal de enfermería tiene un papel preponderante en el cuidado de la salud, es quien acompaña y sigue a cada paciente a lo largo de la evolución de su enfermedad, tanto en instituciones hospitalarias como en el hogar, les da consejos a pacientes y familiares sobre el cuidado y autocuidado que deben tener y sobre la importancia de la modificación de sus estilos para su salud.

Sin embargo, en algunos países, la responsabilidad del profesional de enfermería va mucho más allá. La prescripción de los medicamentos por el personal de enfermería si bien es una disposición novedosa en México, no lo es en el resto del mundo dado que países como España, Australia, Canadá, Estados Unidos, Suecia, Nueva Zelanda y Reino Unido ya cuentan los Licenciados en Enfermería con esta 
autorización. Este nuevo reto para la enfermería, abre nuevos espacios en la práctica profesional de los Licenciados en Enfermería al prescribir medicamentos del cuadro básico. Así, con la preparación teórica y práctica en farmacología los profesionales de enfermería podrán cumplir cabalmente con la prescripción de los medicamentos para brindar mayor y mejor cuidado a los pacientes. ${ }^{37}$

Lo anterior permite considerar que para ejercer esta disposición de prescripción de los medicamentos, los Licenciados en Enfermería y los pasantes, tendrán que contar con una capacitación y actualización en materia de farmacología, que permita realizar las actividades profesionales seguras y sin riesgo para los pacientes.

Es decir, para lograrse estos resultados tan bien pensados, se hace necesario planificar antes de impartir cada charla, realizar una demostración e incluso, administrar un medicamento, razón por la cual, se hace necesario que los profesionales de enfermería manejen y apliquen los conocimientos referidos a la prevención en hábitos de salud y autocuidados en los pacientes con el fin de disminuir la morbi-mortalidad, conocimientos que van implícitos dentro de la materia de farmacología incluida en su plan de estudios de pre-grado.

\section{Conclusiones}

La seguridad del medicamento no puede ser un proyecto a largo plazo, ha de ser un desafío constante en cada uno de los profesionales que tienen a cargo la vida del paciente, y que toca con sus manos el medicamento para ser aplicado a un individuo con el objetivo de mejorar su salud y calidad de vida.

Sirvan estas reflexiones como acicate a cada enfermero para mantener un vínculo constante con el conocimiento y poder ser fieles cumplidores del concepto de enfermería.

Los farmacéuticos clínicos, expertos en farmacoterapia, pueden desempeñar un papel clave en la mejora de la educación farmacológica y en aumentar la confianza de los estudiantes de enfermería (y de medicina) en su capacidad para seleccionar y administrar la terapia adecuada. Somos, en gran medida, los responsables del diseño e impartición de cursos de formación, especialización y seminarios de educación continua en la rama de la farmacología, dirigido al personal de enfermería, a fin de fortalecer las políticas de formación en la carrera y las políticas sanitarias sobre farmacovigilancia y muy especialmente, para disminuir el margen de error que ya está demostrado.

Un llamado especial a las autoridades de las instituciones de salud, responsables de la creación y cumplimiento de los comités de farmacoterapia y farmacovigilancia, encargados de la detección y reporte de las fallas terapéuticas y reacciones adversas a medicamentos.

\section{Referencias}

1. Sandoval Salinas E, Rodríguez Núñez Y. Nivel de satisfacción del interno(a) de enfermería en su formación profesional. Trujillo, 2014. In Crescendo Ciencias de la Salud, 2017; 4(2): 319-329.

2. Malgor LA, Valsecia ME. Farmacología médica. Capítulo I. Introducción a la Farmacología. Marco conceptual. Definiciones. En: Malgor LA, Valsecia; Farmacología general y del sistema nerviosos autónomo, 1-12 Vol 1. Cátedra de farmacología. Facultad de Medicina. Universidad Nacional del Nordeste. Winters JC. 1993. Disponible en: https://cahuanajohn.files.wordpress.com/2009/06/1 farmacologia-5volumenes.pdf

3. Morón Rodríguez FL, Levy Rodríguez M. Farmacología General Editorial de Ciencias Médicas. Cuba.2002

4. Nava P. La importancia de la Farmacología en Enfermería. 2018. Disponible en: https://www.deustosalud.com/blog/rehabilitacion/farmacologiaen-enfermeria

5. Morán Aguilar V, Mendoza Robles AL. Proceso de Enfermería Uso de los lenguajes NANDA, NIC y NOC. Modelos referenciales. 1a ed. México: Trillas; 2010.

6. Iyer $\mathrm{P}$, Taptich B, Bernocchi D. Proceso de Enfermería y Diagnóstico de Enfermería. México: Mc Graw- Hill Interamericana; 1997. $453 \mathrm{p}$

7. González Pisano MM, Mirón Ortega D, Cotiello Cueria Y, Peñacoba Maestre D, Iglesias Rivero RM, Arias Barrientos S. Guía de metodología y diagnósticos de enfermería. Grafinsa. Oviedo. 2002. p 17-48

8. Sosa Hernández ML. Capítulo I. Generalidades. Farmacología y el Proceso de Atención de Enfermería (PAE). En: Vergel Rivera GM, Tasé Martínes MJ, Groning Roque E. Farmacología. Proceso de Atención de Enfermería. 1a ed. La Habana, Cuba: Editorial Ciencias Médicas; 2009. p. 1-5

9. Aristil Chéry PM. Capítulo 4. Vía de administración de los medicamentos. En: Aristil Chéry PM. Manual de farmacología básica y clínica. 62 ed. 2014

10. Carmona Torres JM, Baena Serrano AM, Berral Jiménez AC, Sotelo Baena Q, Recio Andrade B, Recio Andrade I. Análisis de conocimiento sobre la administración de fármacos. European Journal of Investigation in Health, Psychology and Education. 2015;5(2):233-241.

11. Tovar-Riveros BE, Saavedra-Vanegas GG. Problemática en e aprendizaje vs manejo y administración de medicamentos. Ciencia y Humanismo en la Salud. 2017; 4 (1):15-22.

12. Perera Días E, Rodríguez Rivera L, Ranero Aparicio V. Reacciones adversas medicamentosas (RAM) en personas mayores. Gerolnfo. [Internet] 2017; [citado 2018 jun 4];12(1):1$22 . \quad$ Disponible en: http://www.medigraphic.com/pdfs/geroinfo/ger-2017/ger171d.pdf

13. García Milián AJ, Galindo Reymod K, Morales Pérez M, León Cabrera P. Farmacovigilancia hospitalaria. Rev Cubana Oftalmol. 2016; 29(4):688-695.

14. Montero Vizcaíno Y, Izquierdo Santa Cruz M, Vizcaino Alonso Md, Montero Vizcaino YY. Conocimiento del manejo práctico de fármacos en Enfermería. Servicio de reanimación neonatal. Hospital Ginecobstétrico Guanabacoa. Rev haban cienc méd. 2017;16(5):822-831.

15. Barbosa Felipe AO, Borges de Oliveira M, de Souza Terra F. Conocimiento del equipo de enfermería que trabaja en Unidades Pediátrica y Neonatal sobre reacciones adversas a medicamentos. Enferm. glob. 2014; 13(35): 1-10.

16. Farmacovigilancia. En Ecuador se empieza a implementar la farmacovigilancia. [Internet] 2012 [citado 2017 dic 12]. Disponible en: http://farmacovigilancia.tv/blog/en-ecuador-seempieza-a-implementar-la-farmacovigilancia/

17. Samaniego E. Principios básicos: concepto, división e importancia de la Farmacología. El medicamento como instrumento social. Relación con otras ciencias. Desarrollo histórico. En: Fundamentos de Farmacología Médica. T-I. 8va Edición. Editorial Universitaria de la UCE, Ecuador, 2014; 1-19

18. Sheikh A, Dhingra-Kumar N, Kelley E, Kieny MP, Donaldson LJ. El tercer reto mundial porla seguridad del paciente: reducir los daños causados por los medicamentos. [Internet] 2018 [citado 2018 jun 4]. Disponible en: http://www.who.int/bulletin/volumes/95/8/17-198002/es/

19. Consejo Nacional de Salud. Comisión Nacional de Medicamentos e Insumos. Cuadro Nacional de Medicamentos Básicos y Registro Terapéutico [Internet]. 2014 [citado 2018 ene 17]. Disponible en: http://www.conasa.gob.ec/phocadownload/cnmb9na/Cuadro_Na cional_de_Medicamentos_Basicos_9na_Revision.pdf

20. Araujo López DA. Fármaco-nutrimento: interacción insuficientemente considerada. Rev Esp Méd Quir 2014;19:244250. 
21. Feitosa Formiga LM, Mendes de Freitas R, de Oliveira Lima LH, Nascimento de Sousa LS, Feitosa Formiga RC, Silveira Macedo D. Interacción con otros medicamentos: el conocimiento del personal de enfermería en un hospital público. Rev Enferm UFPI. 2014 Oct-Dec;2(4):18-26.

22. Bond C, Raehl C, Franke T. Clinical Pharmacy Services, Hospital Pharmacy Staffing, and Medication Errors in the United States Hospitals. Pharmacotherapy. 2002;22(2):134-47.

23. National Coordinating Council for Medication Error Reporting and Prevention. NCCMERP Taxonomy of medication errors, 1998. Disponible en: http://www.nccmerp.org/about-medication-errors

25. Otero MJ, Martín R, Robles MD, Codina C. 2.14.Errores de medicación. En: Farmacia Hospitalaria. 713-747. 2002

26. Otero MJ, Domínguez-Gil A. Acontecimientos adversos por medicamentos: una patología emergente. Farm Hosp 2000; 24:258-66.

27. National Coordinating Council for Medication Error Reporting and Prevention. NCCMERP Index for categorizing medication errors, 2001

$$
\text { Disponible }
$$
http://www nccmerp org/medErrorCatlndex.htm

28. Vaca Aúz AY, Muñoz Navarro ZP, Flores Grijalba MC, Altamirano Zabala GN, Meneses Dávila MS, Barahona Cisneros $\mathrm{ME}$, et al. Eventos adversos relacionados con los cuidados de enfermería en el Hospital de Ibarra, provincia de Imbabura. Enfermería Investiga, Investigación, Vinculación, Docencia y Gestión. 2016;1(3):102-106

29. Páez Arellano TG, Meneses Dávila MS, Hidrobo Guzmán JF, Jaramillo Villarruel DE, Alvarez Moreno MM. Errores en la prescripción y manejo de la medicación en el Servicio de Pediatría del Hospital San Vicente de Paúl, Ibarra, 2016. Enfermería Investiga, Investigación, Vinculación, Docencia y Gestión. 2016;1(3):98-101.

30. Cuadros Carlesi K, Grillo Padilha K, Toffoletto MC, HenriquezRoldán C, Canales Juan MA. Ocurrencia de Incidentes de
Seguridad del Paciente y Carga de Trabajo de Enfermería. Rev. Latino-Am. Enfermagem. 2017;25:e2841.

31. Universidad Europea. Humantern UEM. Plataforma colaborativa Terminología plurilingüe en el ámbito humanitario. Disponible en: http://www.humantermuem.es/tiki-

index.php?page=farmacolog\%C3\%ADa+cl\%C3\%ADnica

32. Riquelme Pérez M. Educación para la salud escolar. En: AEPap ed. Curso de Actualización Pediatría 2006. Madrid: Exlibris Ediciones; 2006 . p. 185-6. Disponible en: https://www.aepap.org/sites/default/files/educacion_salud_escue la.pdf

33. Consejo Internacional de Enfermeras. La definición de Enfermería. 2017 Disponible en: http://www.icn.ch/es/who-weare/icn-definition-of-nursing/

34. OMS. Enfermería. http://www.who.int/topics/nursing/es/

Disponible en:

35. Medical Assistan. ¿Cuáles son las enfermedades más comunes en los adultos jóvenes? [Internet] 2018 [citado 2018 jun ] Disponible en: https://medicalassistantsalud.com/cuales-son-lasenfermedades-mas-comunes-en-los-adultos-jovenes

36. OMS. Envejecimiento y salud. [Internet] 2018 [citado 2018 jun 4] Disponible en: http://www.who.int/es/news-room/factsheets/detail/envejecimiento-y-salud

37. Valderrama-Sanabria ML. Intervención de enfermería en la administración de medicamentos en pediatría. Rev. cienc. cuidad. 2018;15(1): 72-81

38. Balseiro Almario L, Osuna E, Javier Cabrera D. La prescripción de los medicamentos por los licenciados en enfermería: implicaciones de responsabilidad legal. Revista Conamed. 2017; 22(3):145-151.

Enferm Inv. 3(1):95-104 